\title{
Transient Palsy of Recurrent Laryngeal Nerve Postresection of Giant Substernal Goiter
}

\author{
Rwakaryebe Muhoozi $^{1}$ Fenglei Yu ${ }^{1}$ Jingqun Tang ${ }^{1}$ Xiang Wang ${ }^{1,2}$ \\ ${ }^{1}$ Department of Cardiothoracic Surgery, Second Xiangya Hospital of \\ Central South University, Changsha, Hunan, China \\ 2 Key Laboratory of Carcinogenesis and Cancer Invasion, Cancer \\ Research Institute, Xiangya School of Medicine, Central South \\ University, Changsha, Hunan, China \\ Thorac Cardiovasc Surg Rep 2014;3:51-54.

\begin{abstract}
Address for correspondence Xiang Wang, MD, PhD, Department of Cardiothoracic Surgery, Second Xiangya Hospital of Central South University \& Key Laboratory of Carcinogenesis and Cancer Invasion, Cancer Research Institute of Central South University, 139 Renmin Road, Changsha, Hunan Province 410011, China (e-mail: wangxiang@csu.edu.cn).
\end{abstract}

\begin{abstract}
Keywords

- mediastinum

- recurrent laryngeal nerve

- substernal goiter

We report a case of a female patient aged 46 years with a history of nodular goiter for which she had a subtotal thyroidectomy 31 years ago. She was referred to the emergency department of our hospital because of dyspnea and chest pain for 20 days, then developed cyanosis and edema of the head and upper extremities. Chest $\mathrm{X}$-ray revealed tracheal repulsion. Cervical and thoracic computed tomography showed a giant solid and a cystic mass in the anterior mediastinum and bilateral pleural effusion. The neck ultrasound did not show any thyroid masses. An exploratory thoracotomy with extensive resection considering the anatomical relation of the mass and the adjacent structures was planned. Immediately after the operation, the patient developed airway complications that resolved in 7 days. The tumor was confirmed pathologically as nodular goiter. The overall outcome of the patient was positive; she is healthy after more than 12 months of follow-up. This report examines the approach to diagnosis and management of one of the most common surgical complication associated with substernal goiters.
\end{abstract}

\section{Introduction}

Extension of the goiter beyond the confines of the neck into the thorax with more than $50 \%$ of the mass inferior to the thoracic inlet is called substernal goiter. Herein, we report a case of a 46-year-old female who presented with cyanosis and facial and upper extremities edema. Radiological examination and computed tomographic (CT) scan revealed solid giant mediastinal mass with multiple cystic formations. The neck ultrasound performed before surgery did not show any mass. Considering the radiological images of the solid mass with cystic formation, the differential diagnosis included benign masses such as teratoma. Complete excision was achieved where it was observed that the intrathoracic mass guarded a connection with the cervical thyroid gland, a detail that was not visible on imaging and ultrasound. Histopathology confirmed a nodular goiter.

\section{Case Report}

A 46-year-old female patient with history of nodular goiter for which she had a subtotal thyroidectomy 31 years ago presented to our hospital complaining of dyspnea and chest pain for 20 days; the patient developed cyanosis and edema of the head and upper extremities. Physical examination revealed percussion dullness and wet rales in both hemithorax with decreased breath sounds in the left lung. Blood gas analysis showed moderate oxygenation, $\mathrm{pH} 7.44, \mathrm{pCO}_{2} 49 \mathrm{~mm} \mathrm{Hg}, \mathrm{pO}_{2}$ $48 \mathrm{~mm} \mathrm{Hg}$, and $\mathrm{SO}_{2} 84 \%$. Laboratory examination revealed leukocytosis. Chest $\mathrm{X}$-ray revealed cervicothoracic radiopacity received

October 24, 2013

accepted after revision

December 11, 2013

published online

February 27, 2014
DOI http://dx.doi.org/

10.1055/s-0034-1368099. ISSN 2194-7635. (c) 2014 Georg Thieme Verlag KG
Stuttgart · New York

License terms

(1) $\circledast \circledast$ 


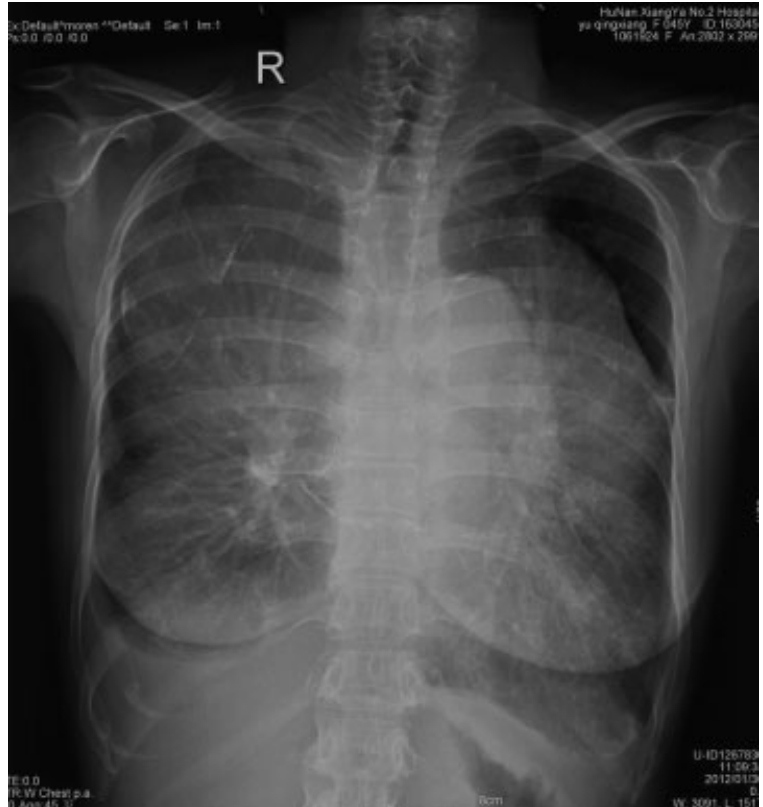

Fig. 1 Chest X-ray revealing cervicothoracic radiopacity and widened mediastinum with tracheal repulsion.

with tracheal repulsion ( $\mathbf{- F i g} . \mathbf{1}$ ). Cervical and thoracic CT scans showed a giant solid and cystic mass in the anterior mediastinum from the thoracic inlet extending to the pulmonary trunk and bilateral pleural effusion ( - Fig. 2) The neck ultrasound did not show any thyroid abnormalities or connection of the intrathoracic mass to the cervical thyroid gland. Biopsy of the mass was contraindicated, considering the close proximity of the tumor and the great vessels in the anterior mediastinum with already clinical manifestation of superior vena cava (SVC) syndrome and imaging showing complete enclosure of the left innominate vein.

\section{Operation Procedure}

Exploratory thoracotomy was performed via a median sternotomy. A highly vascularized giant tumor was exposed in the anterior mediastinum ( - Fig. 3 ), with lots of varicose vessels seen on the tumor surface. The innominate vein and the SVC were not accessible, as the former was englobed by the mass, presenting no obvious interface for dissection. Complete tumor excision was achieved by blunt dissection of the lower and rear margin of the tumor after the varicose vessels were ligated at its edges. We ligated all the afferent blood vessels to the tumor to minimize blood loss that would occur during the resection. To expose the lower and rear part of the tumor, we had to lift the tumor from the bottom which resulted into reduced radial arterial pressure, as the upper part of the tumor compressed the aortic arch; however, the tumor was quickly enucleated after resecting the left innominate vein which was englobed by the tumor. All this time we were providing extra care to protect the recurrent laryngeal nerve (-Fig. 4). Gauze packing and cauterization at the tumor bed successfully stopped the minimal bleeding from the tissues that comprised the tumor bed. We collected $2,500 \mathrm{~mL}$ of blood from the surgical site, and autologous blood transfusion was used to retransfuse the blood. Total operation time was 7 hours and postoperative pathology confirmed nodular goiter.

\section{Postoperative Events}

The patient was initially maintained on mechanical ventilation support for 40 hours postoperation. Moments after

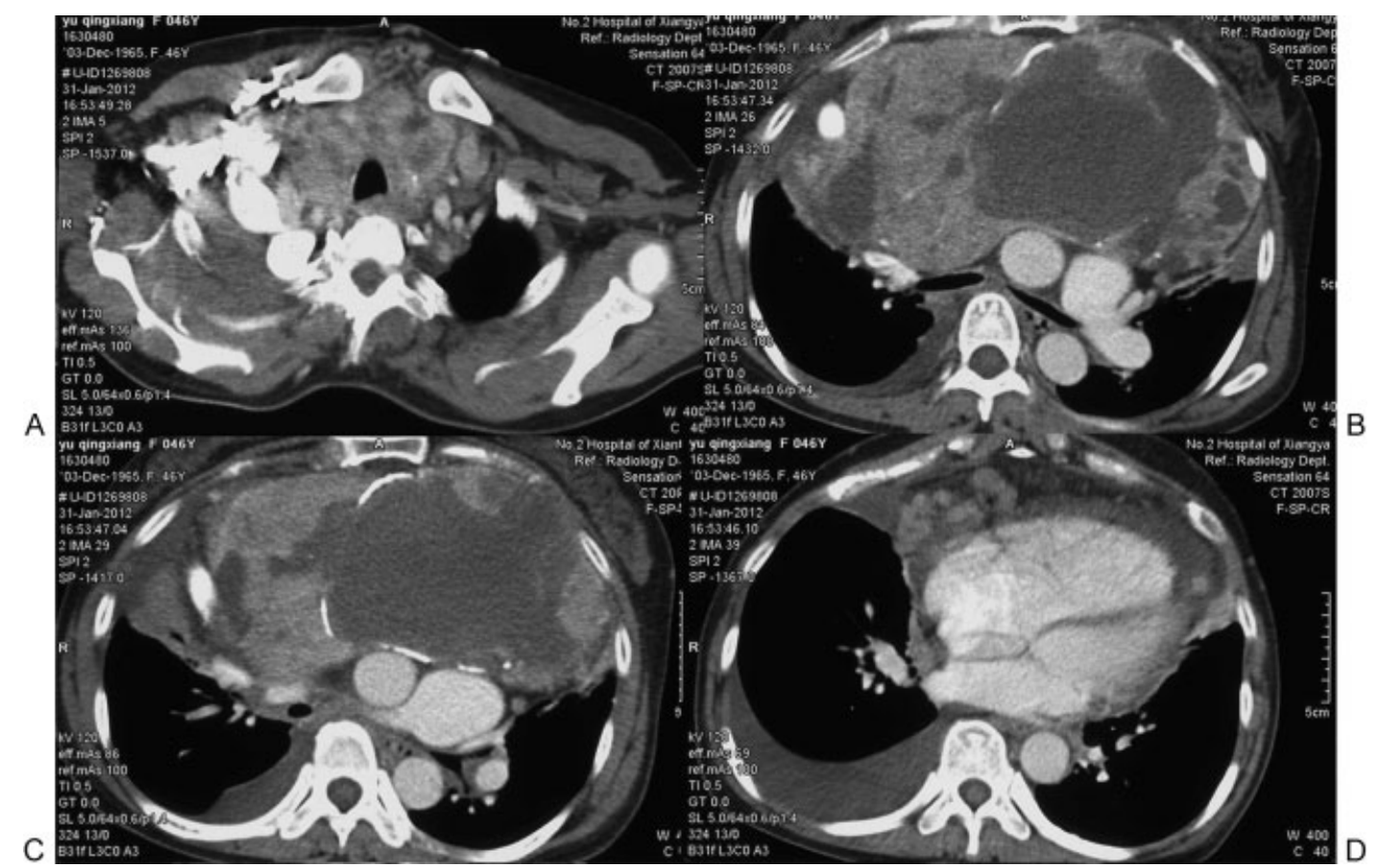

Fig. 2 Computed tomographic scan: Panel A showing no evident connection between the cervical thyroid and the substernal goiter. Panels B and Cillustrating the giant mass with high vascularization patterns that could easily be confused as calcifications. Panel D showing the pleural effusion caused by the tumor. 


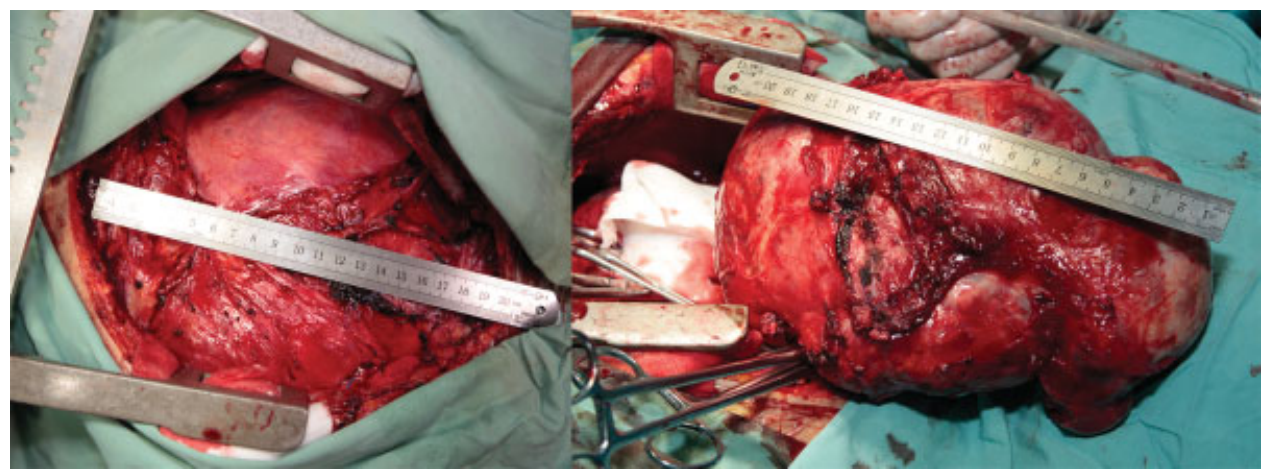

Fig. 3 Intraoperative exposure of the tumor.

extubation, she started presenting respiratory difficulties and lost her voice, and thus an emergency tracheostomy was performed. Bronchoscopy showed the carina, and left and right main bronchi had slightly narrowed, but no bronchomalacia was evidenced. Concerned about a possible recurrent nerve injury, the patient was administered glucocorticoids and nerve nutrition therapy. Mechanical ventilation support was continued for 3 more days through tracheostomy tube, and tracheostomy was closed after 7 days when dyspnea had resolved. She regained her normal voice after closure of tracheotomy stoma. The patient has been followed up for a period of more than 12 months and she is currently healthy.

\section{Discussion}

Substernal goiters are classified as either primary or secondary. The majority of substernal goiters are secondary. They originate from the downward extension of the gland along the planes of the cervical and mediastinal fascia. The connection between the mediastinal and the cervical parts of the gland is usually apparent on imaging or during surgery like in the case of the patient. ${ }^{1}$ Most intrathoracic goiters are located in the anterior mediastinum in front of the subclavian and

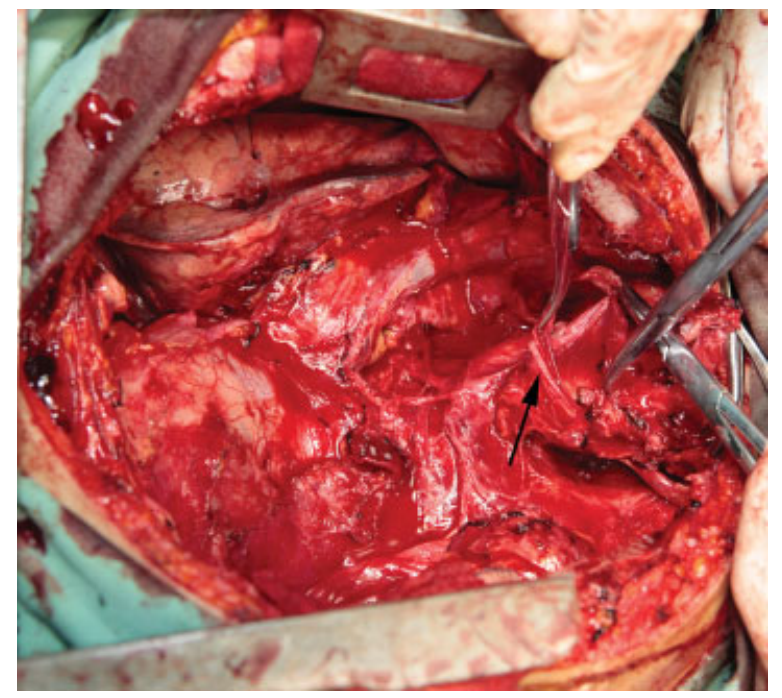

Fig. 4 Operative picture showing the recurrent laryngeal nerve, note the care taken to isolate the nerve. The black arrow showing the recurrent laryngeal nerve adjacent to the tumor bed. innominate vessels, which explains their clinical signs and symptoms of superior vena cava syndrome. This close proximity to such vascular structures also make their resection more complex with increased risk of bleeding and injury that can come to adjacent structures in the anterior mediastinum. ${ }^{2}$ Patients with substernal goiters are asymptomatic for a long period of time until they debut with symptoms associated with compression of intrathoracic structures that usually necessitate surgical treatment unless surgery is contraindicated. This patient had manifestations of airway obstruction and SVC syndrome due to trachea deviation and vascular compression, respectively. These two features are pathognomonic of giant substernal goiters with considerable time of clinical evolution. ${ }^{3}$ With the patient's clinical history, the presenting symptoms, and the imaging studies showing a giant mass in the anterior mediastinum, it was obviously a substernal goiter, however, not evidently showing its connection with the cervical gland. Airway complications after resection of substernal goiters are common and are related to patient factors among others. There are three basic predictors of airway complications, which are old age, larger goiters, and preoperative evidence of tracheal compression on imaging. ${ }^{4}$ Preoperative chest X-ray of the patient clearly revealed tracheal deviation and bronchoscopy showed narrowing of the left and right bronchi. In this case, the patient had two of the three predictors, the large size of the goiter and the preoperative chest X-ray showing tracheal compression. Furthermore, Shen et $\mathrm{al}^{4}$ discussed the predictors of airway complications including the incision of choice in relation to the size of the tumor. A cervical approach is always recommended for the exploration of the thyroid gland which renders less potential harm to the recurrent laryngeal nerve. In cases where the size of the substernal goiter is enormous and would not pass through the thoracic inlet, a sternotomy is the approach of choice, but it increases the risk of injuring the recurrent nerve. The left recurrent laryngeal nerve has a long course, making it susceptible to damage at various sites. In the thoracic cavity, the nerve can be damaged by surgical manipulation in the resection of the tumor. Steurer et al discussed the mechanisms of injury to the nerve to include complete or partial transection, traction, or handling of the nerve, contusion, crush, burn, clamping, misplaced ligature, and compromised blood supply. Airway complication due to injury of the recurrent nerve is witnessed immediately after the patient is 
extubated. Depending on the extent and the injured section of the nerve, this can cause unilateral or bilateral collapse of the vocal cords. ${ }^{5,6}$ Deliberate identification and carefully tracing the path of the recurrent laryngeal nerve have been reported to minimize the risk of injury to the nerve; we did this as illustrated in -Fig. 4. The permanent lesion of an injured recurrent laryngeal nerve manifests as an irreversible dysfunction of phonation with irreversible respiratory difficulties. The patient's airway complications had resolved by day 7 postoperatively. Therefore, we report a case of transient palsy of the recurrent laryngeal nerve attributed to nerve edema developed during surgical manipulation in the resection of the substernal goiter that gradually resolved, and the patient regained her normal voice and cessation of breathing difficulties.

\section{Conclusion}

Intrathoracic goiter itself is an indication for surgery, and such tumors are best resected by separating the tumor along the membrane and removed as a whole rather than partitioning the tumor which would increase blood loss from highly vascularized tumors. Autologous blood transfusion is recommended in scenarios where malignancy is not suspected.

In any thyroid gland surgery, care of the recurrent nerve is required and usually recommended that the nerve be identified and isolated before resection is started.

Considering patient' characteristics such as the size of the tumor resected and preexisting trachea deviation due to the tumor, the use of glucocorticoids such as methylprednisolone and dexamethasone before extubation is beneficial in minimizing airway complications. Preoperative bronchoscopy should be performed on all substernal goiter patients to rule out tracheal and bronchi stenosis or tracheomalacia.

\section{Conflict of Interest}

None.

\section{References}

1 Cannon CR, Lee R, Didlake R. Management of the substernal goiter: a team approach. J Miss State Med Assoc 2010;51(7):179-182

2 Marcelino M, Nobre E, Conceição J, Lopes L, Vilar H, de Castro JJ. Superior vena cava syndrome and substernal goiter. Thyroid 2010; 20(2):235-236

3 Erbil Y, Bozbora A, Barbaros U, Ozarmağan S, Azezli A, Molvalilar S. Surgical management of substernal goiters: clinical experience of 170 cases. Surg Today 2004;34(9):732-736

4 Shen WT, Kebebew E, Duh QY, Clark OH. Predictors of airway complications after thyroidectomy for substernal goiter. Arch Surg 2004;139(6):656-659, discussion 659-660

5 Ramadan HH, Wax MK, Avery S. Outcome and changing cause of unilateral vocal cord paralysis. Otolaryngol Head Neck Surg 1998; 118(2):199-202

6 Steurer M, Passler C, Denk DM, Schneider B, Niederle B, Bigenzahn W. Advantages of recurrent laryngeal nerve identification in thyroidectomy and parathyroidectomy and the importance of preoperative and postoperative laryngoscopic examination in more than 100 nerves at risk. Laryngoscope 2002;112(1):124-33 